\title{
In-fiber Single Polarization Diffraction Grating based on Radiant Tilted Fiber Grating
}

\author{
Huabao QIn, ${ }^{1}$ QINYUn He, ${ }^{1}$ ZhIKUn XING, ${ }^{1}$ XI GUO, ${ }^{1}$ ZhIJUN YAN, ${ }^{1,2 *}$ QIZHEN \\ Sun, ${ }^{1,2}$ ChaO WANG, ${ }^{4}$ KaIMING ZhOU, ${ }^{3}$ Deming LIU ${ }^{1}$ And Lin ZhANG ${ }^{3}$ \\ ${ }^{1}$ The School of Optical and Electronic Information, NGIA, Huazhong University of Science and Technology, Wuhan 430074, Hubei, P. R. China \\ ${ }^{2}$ Wuhan National Laboratory for Optoelectronics (WNLO), Huazhong University of Science and Technology (HUST), 430074 Wuhan, China \\ ${ }^{3}$ Aston Institute of Photonic Technologies, Aston University, Birmingham, UK, B4 TET \\ ${ }^{4}$ School of Engineering and Digital Arts, University of Kent, Canterbury, CT2 7NT, UK \\ *Corresponding author: yanzhijun@gmail.com
}

Received XX Month XXXX; revised XX Month, XXXX; accepted XX Month XXXX; posted XX Month XXXX (Doc. ID XXXXX); published XX Month XXXX

In this letter, we proposed an in-fiber single polarization diffraction grating based on a radiant tilted fiber grating (TFG), in which the s-polarization light could be diffracted from the fiber core to free space with the wavelength-dependent diffraction angle. For the first time, we have presented the theoretical model of angular dispersion of radiant TFG by employing Fourier optics analysis method. The angular dispersion of radiant TFG has been numerically characterized in terms of tilt angle, period and wavelength. In the experiment, we have measured the diffraction angles and angular dispersion of the radiant TFGs with tilt angles of $41^{\circ}, 45^{\circ}$ and $47^{\circ} \mathrm{UV}$ inscribed into single mode fiber, where the experimental results matched well with the simulation results. The simulation and experimental results have indicated that the diffraction light of radiant TFGs has a linear polarization state with over 0.99 degree of polarization at the tilt angle range from $41^{\circ}$ to $47^{\circ}$. (C) 2018 Optical Society of America

http://dx.doi.org/10.1364/OL.99.099999

Optical imaging technique is an important and powerful technology for visually designing and fabricating the micro/nanostructure devices, exploring the components of the artificial materials, investigating the internal structure and characteristics of matter [1-3]. Most of the spectrum-based imaging systems employ a diffraction grating to separate the polychromatic light and stretch the laser pulse. Recently, due to the small size, high flexibility, foldable light path features, optical fiber has been widely applied in optical imaging systems as the transmission probe and the imaging systems are gradually transforming from the volume structure to the fiber-based structure [1-6]. So far, the traditional diffraction gratings are mainly based on the bulk optical component, and the complex collimating modules are demanded to align the light transmitting from the fiber in the optical system. The structural difference and the complex collimating modules would result in the inevitable defects of the systems, such as the extra cost, energy loss, complicated structure and increasing the instability of the system, and have limited the further development for system integrations. The desire of a new type of diffraction grating with inherently compatible with the fiber-based system is becoming more and more urgent.

Optical fiber grating is a special kind of in-fiber diffraction device, which could diffract the light between fiber core mode and cladding/radiation mode. So far, the fiber gratings have been widely utilized in fiber sensing, fiber laser and fiber communication areas. In particular, the gratings with blazed structure called tilted fiber gratings (TFGs) are attracting more and more attentions [7-10]. In 2003, K. Feder et. al. have proposed the TFGs with small tilt angles as dispersive elements to achieve spectrum analyzing function [11-12], however, due to that the diffracted light was still restrained in the fiber cladding, the indexmatching prism or oil need to be employed to eliminate the reflection and tap the light out of fiber, which is not convenient for packaging and the diffracted light is not strongly polarization dependent. In the previous works, a $45^{\circ} \mathrm{TFG}$ based ideal polarizer has been proposed, which could directly tap the s-polarization light out of fiber [13-14]. Moreover, our recent works have demonstrated that $45^{\circ} \mathrm{TFG}$ could be an in-fiber dispersive element with high diffraction efficiency in the applications of spectrally encoded imaging, OCT spectrometer and wireless optical communication systems [15-17]. Plenty of papers about the characteristics and applications of fiber gratings have been published, but most of them were focused on the transmission property of fiber gratings. Only a few papers have reported the radiation property of TFGs [18-20], however, so far, there is not any theoretical model to analyze the diffraction property of radiant TFGs. In this letter, we have built the numerical model of the diffraction property of radiant TFG by employing Fourier optics method and measured the angular dispersion of TFGs in the 
experiment. The theoretical and experimental results have shown that the radiant TFGs with tilt angle from $41^{\circ}$ to $47^{\circ}$ could achieve the single polarization diffraction (the grating tilted at $45^{\circ}$ has the strongest diffraction efficiency), which could be potentially applied in polarization sensitive spectra analysis system.

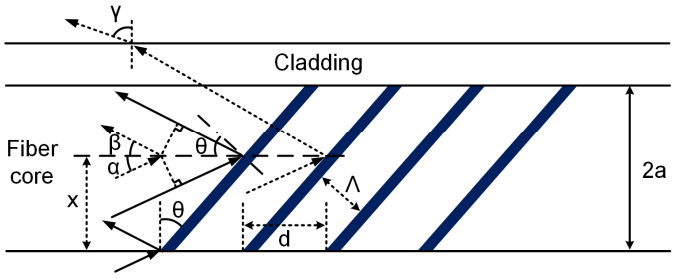

Fig. 1. Diagram for analyzing the diffraction characteristics of TFG.

The grating equation describes the dispersion characteristics of the volume diffraction grating. However, it is not suitable to analyze the in-fiber diffraction grating, due to its core/cladding/air fiber structure and the longitudinally distributed grating structures. In this letter, the Fourier optics analysis method [21] was employed to derive the theoretical model and explain the diffraction of radiant TFGs. As shown in Fig. 1, due to the slight difference of refractive index in the grating pattern, the incident light would be coupled and reflected at the interface of fiber grating. The interactions between the reflected lights from the same and different interfaces would result in the interference and diverge the propagation direction of the light. If the position of the incident light is assumed at $x$ with an angle of $\alpha$, the phase difference between the lights from the same interface could be written as:

$$
\left\{\begin{aligned}
\varphi(x) & =\frac{2 \pi n}{\lambda} \cot \theta(\cos \alpha+\cos \beta) x=2 \pi \xi x \\
\xi & =\frac{n \cot \theta(\cos \alpha+\cos \beta)}{\lambda}
\end{aligned}\right.
$$

where $n$ is the refractive index of the fiber core, $\theta$ is the tilt angle of the grating, $\beta$ is the radiated angle of the light and $\lambda$ is the wavelength of the incident light. The reflection function of the grating could be expressed as:

$$
r(x)=e^{j \varphi(x)} \operatorname{rect}\left(\frac{x}{2 a}\right) * \sum_{n=1}^{N} \delta(x-(n-1) d)(1-\eta)^{n-1}
$$

here, $\eta$ is the attenuation coefficient introduced by the interface reflection and $d=\Lambda / \cos \theta$ is the normal period of the grating. If the incident light field is considered to be $E=e^{j 2 \pi u_{0} x}$, where $u_{0}=n \sin \alpha / \lambda$, the complex disturbance of the diffraction light $U^{\prime}(x)$ would be the Fourier transform of the complex disturbance at the grating planes, which is given by:

$$
\begin{aligned}
U^{\prime}(x)= & C \cdot \mathcal{F}\{r(x) \cdot E\}=C \cdot \mathcal{F}\left\{e^{j \varphi(x)}\right\} * \mathcal{F}\left\{\operatorname{rect}\left(\frac{x}{2 a}\right)\right\} . \\
& \mathcal{F}\left\{\sum_{n=1}^{N} \delta(x-(n-1) d)(1-\eta)^{n-1}\right\} * \mathcal{F}\left\{e^{j 2 \pi u_{0} x}\right\} \\
= & C \cdot \frac{\sin \left(2 a\left(u-u_{0}-\xi\right)\right)}{u-u_{0}-\xi} \cdot \frac{1-(1-\eta)^{N} e^{-j 2 \pi N d\left(u-u_{0}\right)}}{1-(1-\eta) e^{-j 2 \pi d\left(u-u_{0}\right)}}
\end{aligned}
$$

According to Eq. 3, the maximum intensity is determined by $u-u_{0}-\xi=0$ and $u-u_{0}=m / d$, where $m= \pm 1, \pm 2, \ldots$. For a core-cladding structure of optical fiber, the light is not only diffracted by the grating, but also refracted at the interface of cladding and air, which would further enhance the dispersion of radiant TFG. Based on that, the diffraction angle of the radiant TFG could be given by:

$$
\gamma=\arcsin \frac{n \cdot\left(\cot \theta-\frac{n \cdot \Lambda}{\lambda \cdot \sin \theta}\right)}{\sqrt{1+\left(\cot \theta-\frac{n \cdot \Lambda}{\lambda \cdot \sin \theta}\right)^{2}}}
$$

Here, $\gamma$ is the angle that the light diffracted out of fiber. Eventually, the angular dispersion for a radiant TFG can be written as:

$$
\begin{gathered}
\frac{d \gamma}{d \lambda}=\frac{n^{2} \cdot \Lambda}{\left(1+\left(\cot \theta-\frac{n \cdot \Lambda}{\lambda \cdot \sin \theta}\right)^{2}\right) \cdot \lambda^{2} \cdot \sin \theta} . \\
\frac{1}{\sqrt{1+\left(1-n^{2}\right)\left(\cot \theta-\frac{n \cdot \Lambda}{\lambda \cdot \sin \theta}\right)^{2}}} .
\end{gathered}
$$
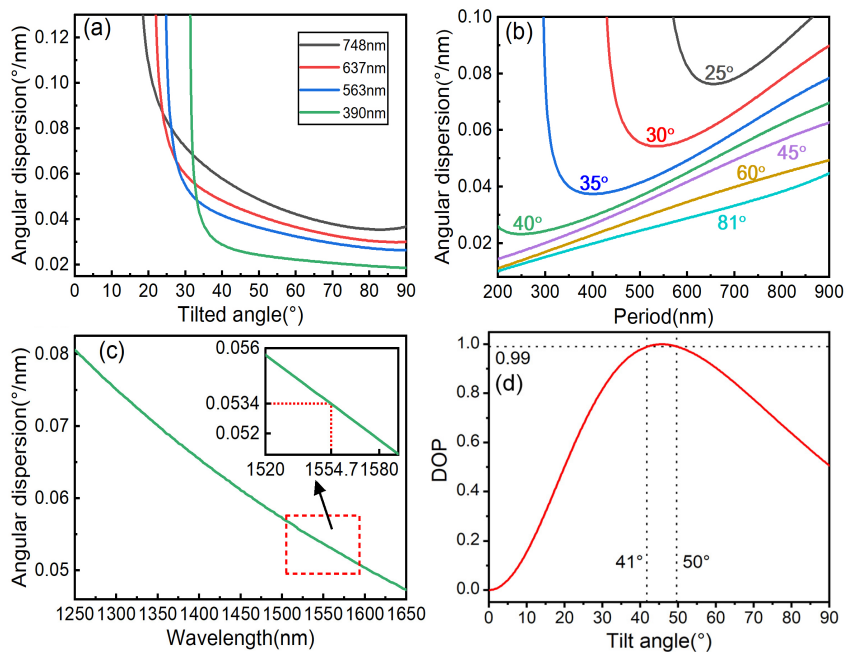

Fig. 2. Simulated results of angular dispersion versus (a) tilt angle at different grating period, and (b) period at 1550nm and (c) working wavelength of TFG; (d) the relationship between degree of polarization (DOP) of the radiated light and the tilt angle.

Equation 5 expresses the angular dispersion of a radiant TFG structure. Based on Eq. 5, we have investigated the angular dispersion characteristics of the radiant TFGs in term of tilt angle, grating period and working wavelength, respectively, and plotted in Fig. 2a, b and c, respectively. As shown in Fig. 2a, the dispersion exhibits a non-linear decrease with respect to the tilt angle at the wavelength of $1550 \mathrm{~nm}$. The rapid fall is caused by the total internal reflection of the fiber and the diffracted angle becomes larger with the smaller tilt angle, which results in the shifting of the turning point of each curve. For the tilt angle above $40^{\circ}$, the grating with a larger period always has a larger dispersion, see in Fig. $2 \mathrm{a}$.

In addition to the tilt angle, the angle dispersion is also related to the period of grating and working wavelength. Fig. $2 \mathrm{~b}$ illustrates 
the relationship between the dispersion and period with different tilted angles at the wavelength of $1550 \mathrm{~nm}$. It is clearly showed that the dispersion curve has a turning point and the corresponding period of the point moves to the smaller period with the increasing of tilt angle, which are the results of the total reflection. The angular dispersion curve is in proportion to the period and consistent with our previous analysis. Therefore, the performance of the in-fiber grating could be enhanced by increasing the grating period, which is totally different from the traditional volume diffraction grating ( $d \theta / d \lambda \sim 1 / d$ ). Meanwhile, in Fig. 2c, we have analyzed the dispersion of TFGs with $748 \mathrm{~nm}$ period versus the wavelength at a tilt angle of $45^{\circ}$, which exhibits a non-linear decrease with respect to the wavelength, where the trend approaches to a small linear change at the range from $1520 \mathrm{~nm}$ to 1590nm (illustrated in Fig. 2c). This characteristic implies that the linear dispersion of grating is almost a constant at this range and the angle difference for a fixed wavelength interval would be a constant as well, which would benefit the design of the detection part of the spectrum analysis system and other applications of the grating. Besides, we have analyzed the degree of polarization (DOP) of diffracted light of $748 \mathrm{~nm}$ period radiant TFG with different tilt angle at $1550 \mathrm{~nm}$, as shown in Fig. $2 \mathrm{~d}$. It is clearly shown that the diffracted light of grating at the tilt angle range from $41^{\circ}$ to $50^{\circ}$ has a DOP over 0.99 , which indicates the perfect single polarization characteristic of the radiant TFGs with $41^{\circ}$ to $50^{\circ}$ tilt angle and it could be an ideal polarization dependent in-fiber dispersive element. The simulation results also show that, although the grating with a smaller tilt angle has a larger dispersion, there is a degradation of DOP with the decrease of tilt angle. In addition, compared with the TFGs with small tilt angles, the grating tilted at $45^{\circ}$ could directly diffract the light out of fiber and achieve the strongest diffraction efficiency.

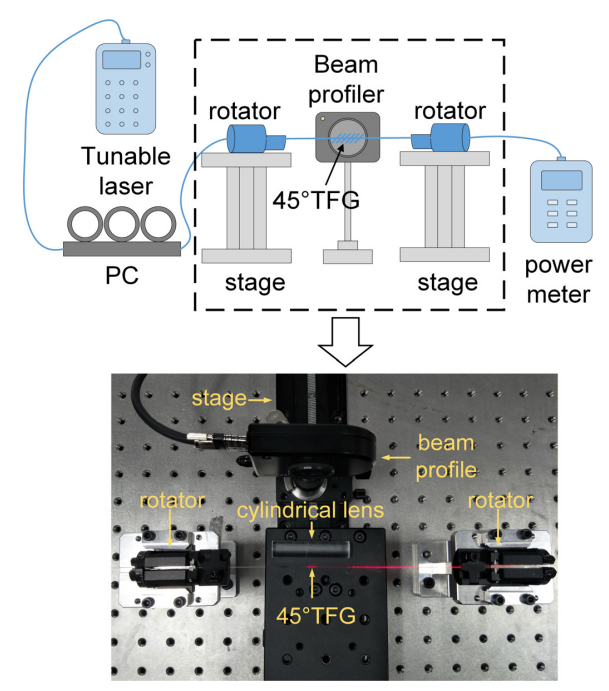

Fig. 3. Diagram and the photograph of the experimental setup for measuring the diffraction angles of TFGs.

To verify our theoretical model, we have experimentally measured the diffraction angles of radiant TFGs with $5 \mathrm{~mm}$ length at the tilt angles of $41^{\circ}, 45^{\circ}$ and $47^{\circ}$, which were UV-inscribed in the single mode fiber by a $1800 \mathrm{~nm}$ phase mask under the same inscription condition. The gratings with the tilt angles of $41^{\circ}, 45^{\circ}$ and $47^{\circ}$ have the period of 792,748 and $728 \mathrm{~nm}$, respectively, and the polarization extinction ratios (PER) are $0.95,6.33$ and $2.3 \mathrm{~dB}$ at $1550 \mathrm{~nm}$, respectively. The diffraction experimental setup is shown in Fig. 3, which is configured with tunable laser (EXFO FLS-2600B), fiber rotators, polarization controller (PC), beam profiler (GentecEO Beamage-4M-IR), 3D-stage and powermeter. To effectively capture the side-tapped light, the light was focused by a cylindrical lens. In the experimental setup, the tunable laser has a $72 \mathrm{~nm}$ wavelength tuning range from $1520 \mathrm{~nm}$ to $1592 \mathrm{~nm}$. The rotators are used to adjust the radiation direction of the radiant TFGs and the beam profiler is employed to capture the radiation patterns and record the spatial position of different incident wavelengths. The PC and powermeter are used to control the polarization state of incident light (the minimum value in powermeter shows that the s-polarized light has been launched into the grating).
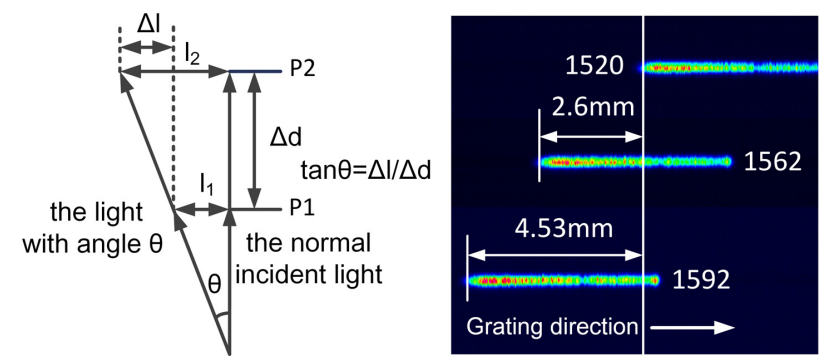

Fig. 4. The measurement principle of the diffraction angle and the captured diffracted patterns of 1520, 1562 and 1592nm light.

The measuring principle is illustrated in Fig. 4. By measuring the deviation $\Delta l=l_{2}-l_{1}$ and $\Delta d$, the diffraction angle could be figured out by the $\arctan$ function $\theta=\arctan (\Delta l / \Delta d)$. In the experiment, we firstly captured the diffracted light from a $45^{\circ} \mathrm{TFG}$ with $748 \mathrm{~nm}$ period at the wavelength of $1520 \mathrm{~nm}, 1562 \mathrm{~nm}$ and $1592 \mathrm{~nm}$, respectively. The normal distance between the grating and CCD was $60 \mathrm{~mm}$. The entire space separation between the wavelengths from $1520 \mathrm{~nm}$ to $1592 \mathrm{~nm}$ is around $4.53 \mathrm{~mm}$ (see in Fig. 4b), which has preliminarily verified the dispersion function of radiant TFG. Furthermore, we have measured the diffraction light from $1520 \mathrm{~nm}$ to $1592 \mathrm{~nm}$ with $4 \mathrm{~nm}$ increment and the results are plotted in Fig. 5a. The black dots are the deviations of the diffraction light measured at different pixel positions, which are perfectly fitted by a linear curve with $\mathrm{R}^{2}=0.9994$. And the normal diffracted wavelength is calculated to be $1554.7 \mathrm{~nm}$. The relationship between the diffraction angle and wavelength is plotted in blue dots. As shown in Fig. 5a, the diffraction angles are linearly shifted with the changing of wavelength and give an angular dispersion coefficient around $0.054^{\circ} / \mathrm{nm}$, which is in excellent accord with the simulated value of $0.053^{\circ} / \mathrm{nm}$. This value is lower than that of the volume grating $\left(0.1083^{\circ} / \mathrm{nm}\right.$ with the period of $748 \mathrm{~nm}$ ), however, it has met the requirements for the most of applications in the spectral imaging and fiber communication systems [15-17]. Furthermore, we have compared the diffraction angles of radiant TFGs with different tilt angles $\left(41^{\circ}\right.$, $45^{\circ}$ and $47^{\circ}$ ), as illustrated in Fig. 5b. The measured angular dispersions are $0.0640,0.0535$ and $0.0496^{\circ} / \mathrm{nm}$, respectively, where the simulation results are $0.0638,0.0534$ and $0.0500^{\circ} / \mathrm{nm}$, respectively. The agreement between measurement and 
simulation results has further verified the applicability of the proposed model for describing the dispersion characteristics of radiant TFGs. The experimental results shown in Fig. 5b have also indicated that the perpendicularly radiated wavelength of gratings fabricated by the same phase mask is shifted with the changing of the tilt angle. In addition, we have measured the polarization distributions of the diffracted light from these three TFGs, see in Fig. 5c. As shown in the figure, the perfect "8" letter distributions indicate that the diffracted lights are linearly polarized. The diffracted lights from the TFGs with tilt angles of $41^{\circ}, 45^{\circ}$ and $47^{\circ}$ have the DOPs of about 0.9908, 0.9968 and 0.9962, respectively. By monitoring the transmitted and radiated light intensity, we have also calculated the diffraction efficiency (DE) of the radiant TFGs, which showed a dependency on the PER of grating. The TFGs with the tilt angles of $41^{\circ}, 45^{\circ}$ and $47^{\circ}$ have around $9.82 \%$, $38.36 \%$ and $20.56 \%$ DE at $1550 \mathrm{~nm}$, respectively, and the $45^{\circ} \mathrm{TFG}$ has a higher DE compared with other tilt angles. The larger of the PER indicates the stronger ability to diffract the light out of fiber. Finally, the experimental results have verified the theoretical model and demonstrated that the radiant TFGs could act as the single polarization in-fiber diffraction devices.
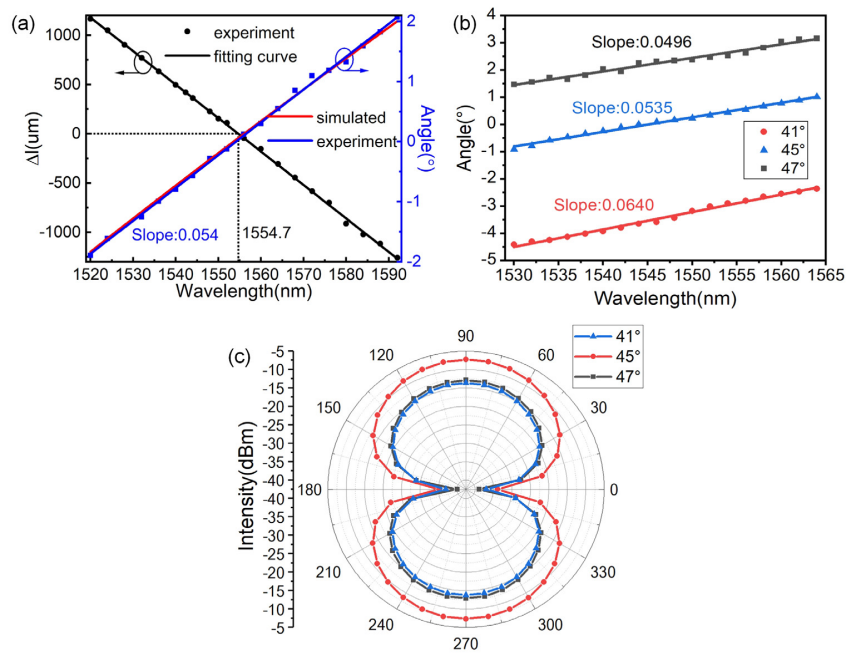

Fig. 5. (a) the simulated and experimental results of diffraction angles; (b) the measured diffraction angles of TFGs with different tilt angles; (c) the measured polarization distributions of the diffracted light.

In conclusion, we have proposed an ideal in-fiber single polarization diffraction grating based on radiant TFGs, which would be potentially applied in polarization sensitive spectral imaging field. Based on Fourier optics analysis, we have firstly derived the diffraction theoretical model of radiant TFGs and experimentally investigated the dispersion characteristics of radiant TFGs with different tilt angles. The simulation results show that the angular dispersion of radiant TFGs is related with the parameters of the grating and the incident light. In the experiment, we have measured the diffraction characteristics of the radiant TFGs with $41^{\circ}, 45^{\circ}$ and $47^{\circ}$ tilt angles, which have the angular dispersions of $0.0640,0.0535$ and $0.0496^{\circ} / \mathrm{nm}$, respectively. All the simulation and experiment results are in good agreement, which has further verified the validity of the proposed diffraction theoretical model. Finally, we have measured the polarization distributions of the diffraction light, which showed that the in-fiber grating could achieve the perfect polarization characteristics in a certain tilt angle range.

Funding. This work is supported by National Natural Science Foundation of China (No. 61505244) and the Science Fund for Creative Research Groups of the Nature Science Foundation of Hubei (NO. 2018CFA004), the Major Projects of Technical Innovation of Hubei (NO. 2018AAA040), the National Key Research and Development Program of China (No. 2018YFB2100902), the Fundamental Research Funds for the Central Universities (No. 2019kfyRCPY095), the Fundamental Research Funds for the Central Universities, HUST: $2017 \mathrm{KFYXJJ033.}$

\section{References}

1. A. A. Gowen, C. P. O'Donnell, P. J. Cullen, G. Downey, and J. M. Frias, Trends Food Sci. Technol. 18, 590-598 (2007).

2. A. M. Zysk, F. T. Nguyen, A. L. Oldenburg, D. L. Marks, and S. A. Boppart, J Biomed. Opt. 12, 051403 (2007).

3. C. Lei, B. S. Guo, Z. Z. Cheng, K. Goda, Appl. Phys. Rev. 3, 011102 (2016).

4. Y. Yasuno, S. Makita, Y. Sutoh, M. Itoh, T. Yatagai, Opt. Lett. 27, 1803-1805 (2002).

5. B. Cense, M. Mujat, T. C. Chen, B. H. Park, and J. F. de Boer, Opt. Express 15, 2421-2431 (2007).

6. H. Wang, T. Akkin, C. Magnain, R. Wang, J. Dubb, W. J. Kostis, M. A. Yaseen, A. Cramer, S. Sakadzic, and D. Boas, Opt. Lett. 41, 2213-2216 (2016).

7. J. Albert, L. Y. Shao, C. Caucheteur, Laser Photonics Rev. 7, 83-108 (2013).

8. T. Guo, F. Liu, B.O. Guan, J. Albert, Opt. Laser Technol. 78, 19-33 (2016).

9. J. Zheng, X. Dong, P. Zu, L. Y. Shao, C. C. Chan, Y. Cui, and P. P. Shum, Opt. Express, 21, 17863-17868 (2013).

10. B. Jiang, K. Zhou, C. Wang, Q. Sun, G. Yin, Z. Tai, K. Wilson, J. Zhao, L. Zhang, Sens. Actuators B Chem. 254, 1033-1039 (2018).

11. K. Feder, P. Westbrook, J. Ging, P. Reyes, and G. Carver, IEEE. Photonics Technol. Lett. 15, 933-935 (2003).

12. J. L. Wagener, T. A. Strasser, J. R. Pedrazzani, J. DeMarco, and D. DiGiovanni, in 23rd ECOC, Conf. Publ 448, pp. 65-68 (1997).

13. Z. Yan, C. Mou, K. Zhou, X. Chen, and L. Zhang, J. Lightwave Technol. 29, 2715-2724 (2011).

14. M. T. Posner, N. Podoliak, D. H. Smith, P. L. Mennea, P. Horak, C. B. Gawith, P. G. Smith and J. C. Gates, Opt. Express, 27, 11174-11181 (2019).

15. G. Wang, C. Wang, Z. Yan, and L. Zhang, Opt. Lett. 41, 2398-2401 (2016).

16. S. Remund, A. Bossen, X. Chen, L. Wang, L. Zhang, B. Považay, and C. Meier, Proc. SPIE 9129, 91293G (2014).

17. G. Wang, U. Habib, Z. Yan, N. J. Gomes, Q. Sui, J. Wang, L. Zhang, and C. Wang, J. Lightwave Technol. 36, 4618-4625 (2018).

18. Y. Li, M. Froggatt, T. Erdogan, J. Lightwave Technol. 19, 1580-1591 (2001).

19. T. Erdogan and J. E. Sipe, Opt. Lett. 20, 1838-1840 (1995).

20. C. Jáuregui and J. López Higuera, Microw. Opt. Techn. Let., 37, 124-127 (2003).

21. J. W. Goodman, Introduction to Fourier Optics (Roberts, 2005). 
1. A. Gowen, C. O'Donnell, P. Cullen, G. Downey, and J. Frias, "Hyperspectral imaging-an emerging process analytical tool for food quality and safety control," Trends in food science technology 18, 590598 (2007).

2. A. M. Zysk, F. T. Nguyen, A. L. Oldenburg, D. L. Marks, and S. A. Boppart, "Optical coherence tomography: a review of clinical development from bench to bedside," J Biomed Opt 12, 051403 (2007).

3. C. Lei, B. S. Guo, Z. Z. Cheng, and K. Goda, "Optical time-stretch imaging: Principles and applications," Appl Phys Rev 3, 011102 (2016).

4. Y. Yasuno, S. Makita, Y. Sutoh, M. Itoh, and T. Yatagai, "Birefringence imaging of human skin by polarization-sensitive spectral interferometric optical coherence tomography," Opt Lett 27, 1803-1805 (2002).

5. B. Cense, M. Mujat, T. C. Chen, B. H. Park, and J. F. de Boer, "Polarizationsensitive spectral-domain optical coherence tomography using a single line scan camera," Opt Express 15, 2421-2431 (2007).

6. H. Wang, T. Akkin, C. Magnain, R. Wang, J. Dubb, W. J. Kostis, M. A. Yaseen, A. Cramer, S. Sakadzic, and D. Boas, "Polarization sensitive optical coherence microscopy for brain imaging," Opt Lett 41, 2213-2216 (2016).

7. J. Albert, L. Y. Shao, and C. Caucheteur, "Tilted fiber Bragg grating sensors," Laser Photon Rev 7, 83-108 (2013).

8. T. Guo, F. Liu, B.O. Guan, and J. Albert, "Tilted fiber grating mechanical and biochemical sensors," Optics Laser Technology 78, 19-33 (2016).

9. J. Zheng, X. Dong, P. Zu, L. Y. Shao, C. C. Chan, Y. Cui, and P. P. Shum, "Magnetic field sensor using tilted fiber grating interacting with magnetic fluid," Opt Express 21, 17863-17868 (2013).

10. B. Q. Jiang, K. M. Zhou, C. L. Wang, Q. Z. Sun, G. L. Yin, Z. J. Tai, K. R. Wilson, J. L. Zhao, and L. Zhang, "Label-free glucose biosensor based on enzymatic graphene oxide-functionalized tilted fiber grating," Sensor Actuat B-Chem 254, 1033-1039 (2018).

11. K. Feder, P. Westbrook, J. Ging, P. Reyes, and G. Carver, "In-fiber spectrometer using tilted fiber gratings," IEEE Photonics Technology Letters 15, 933-935 (2003).
12. J. L. Wagener, T. A. Strasser, J. R. Pedrazzani, J. DeMarco, and D. DiGiovanni, "Fiber grating optical spectrum analyzer tap," in Integrated Optics and Optical Fibre Communications, 11th International Conference on, and 23rd European Conference on Optical Communications (Conf. Publ. No.: 448), (IET, 1997), 65-68.

13. Z. Yan, C. Mou, K. Zhou, X. Chen, and L. Zhang, "UV-inscription, polarization-dependant loss characteristics and applications of $45^{\circ}$ tilted fiber gratings," J. Lightwave Technol. 29, 2715-2724 (2011).

14. M. T. Posner, N. Podoliak, D. H. Smith, P. L. Mennea, P. Horak, C. B. Gawith, P. G. Smith, and J. C. Gates, "Integrated polarizer based on $45^{\circ}$ tilted gratings," Opt Express 27, 11174-11181 (2019).

15. G. Wang, C. Wang, Z. Yan, and L. Zhang, "Highly efficient spectrally encoded imaging using a $45^{\circ}$ tilted fiber grating," Opt Lett 41, 2398-2401 (2016).

16. S. Remund, A. Bossen, X. Chen, L. Wang, L. Zhang, B. Považay, and C. Meier, "Fiber optically integrated cost-effective spectrometer for optical coherence tomography," in Biophotonics: Photonic Solutions for Better Health Care IV, (International Society for Optics and Photonics, 2014), 91293G.

17. G. Wang, U. Habib, Z. Yan, N. J. Gomes, Q. Sui, J. Wang, L. Zhang, and C. Wang, "Highly efficient optical beam steering using an in-fiber diffraction grating for full duplex indoor optical wireless communication," J. Lightwave Technol. 36, 4618-4625 (2018).

18. Y. Li, M. Froggatt, and T. Erdogan, "Volume current method for analysis of tilted fiber gratings," J. Lightwave Technol. 19, 1580 (2001).

19. T. Erdogan and J. Sipe, "Tilted fiber phase gratings," JOSA A 13, 296-313 (1996).

20. C. Jáuregui and J. López - Higuera, "Near - field theoretical model of radiation from a uniform - tilted fiber - Bragg grating," Microw. Opt. Techn. Let., vol. 37, no. 2, pp. 124-127, 2003..

21. Goodman, Joseph W. Introduction to Fourier optics. Roberts and Company Publishers, 64-91, 2005 